\title{
ORAL HEALTH ASSESSMENT IN CHILDREN AND ADOLESCENTS WITH TYPE 1 DIABETES
}

\author{
Mohammad H. Awad ${ }^{*}$, Nasr M. Attia ${ }^{* *}$ and Nanees A. Salem ${ }^{* * *}$
}

\begin{abstract}
Objective: To assess oral health status in relation to diabetes-related variables in a cohort of children and adolescents with type 1 diabetes mellitus (T1DM).

Study design: Eighty-eight children and adolescents with T1DM (median age: 12 years; range: 6-17 years) had received a comprehensive oral health examination performed by a single dentist and included, simplified oral hygiene index (OHI-S), gingival-index (GI), dental caries indices (DMFT and $\mathrm{dft}$ ) and stimulated salivary flow rate (SSFR; $\mathrm{ml} / \mathrm{min}$ ).

Results: Significantly higher frequency of patients had fair oral hygiene and moderate gingivitis $(P<0.001)$. Significantly lower stimulated salivary flow rate was detected in patients within late childhood group $(P=0.038)$. Increased prevalence of permanent dental caries was observed in late adolescent period $(P=0.001)$. There was significant increase in primary dental caries in children diagnosed with diabetes before six years $(P=0.004)$. Patient's age was positively correlated with SSFR, gingival-index and DMFT-index. DMFT-index was positively correlated with T1DM duration and HbA1c level. There was significant positive correlation between OHI-S and GI.

Conclusion: Children and adolescents with T1DM displayed unfavorable oral health profiles that have been strongly linked to patient's age, age at onset and duration of T1DM and level of metabolic control. Regular recall dental visits must be considered.
\end{abstract}

KEY WORDS: Oral health, Type 1 Diabetes Mellitus, Children.

\section{INTRODUCTION}

Type 1 diabetes mellitus (T1DM) is an immunemediated pancreatic $\beta$-cell destruction resulting in either partial or absolute insulin deficiency. It is estimated that around 96000 child is diagnosed with T1DM every year. ${ }^{1}$
The long-term vascular complications of diabetes affect the highly vascular organs; include microvascular complications as nephropathy, retinopathy and neuropathy; and macrovascular complications as cardiovascular and cerebrovascular diseases. $^{2}$

\footnotetext{
* Lecturer, Department of Pediatrics, Endocrinology \& Diabetes Unit, Faculty of Medicine, Mansoura University, Egypt

** Lecturer, Pedodontics and Dental Public Health Department, Faculty of Dentistry, Mansoura University, Egypt

*** Associate professor, Department of Pediatrics, Endocrinology \& Diabetes Unit, Faculty of Medicine, Mansoura University, Egypt
} 
Dental complications are considered one of diabetes-related vascular complications, where similar changes in small vessels can be found in the oral tissues. Vascular complications of diabetes are usually rare to present clinically during childhood and adolescence. However, early functional and structural abnormalities can be detected few years after the onset of diagnosis. Delaying the onset and progression of complications is possible by early intensive education during the childhood and adolescence period. ${ }^{3}$

Children and adolescents with T1DM are at higher risk of developing oral health complications including; dental caries, periodontal disease, gingivitis, oral mucosal infections, and saliva dysfunction. In young children with diabetes, periodontal disease can develop at a younger age than non-diabetics. ${ }^{3}$ Several researches indicated that oral health problems development in children with T1DM is multifactorial. This was explained by Frantzis et al. who considered diabetes as a predisposing factor and not the direct cause for periodontal disease. ${ }^{4}$ The lower resistance in diabetes to oral infections can be attributed to the existing metabolic imbalances, leading to rapid progression of periodontal diseases. In addition, poor glycemic control contributes to reduced salivary flow, high blood and salivary levels of glucose also may lead to increasing risk of dental caries. ${ }^{5,6}$

We aimed to assess oral health status including; oral hygiene, gingival state, dental caries, and salivary flow rate in a cohort of children and adolescents with T1DM and in relation to diabetesrelated factors.

\section{SUBJECTS AND METHODS}

A cross-sectional study conducted between March 2019 and February 2020. One-hundred children aged between 6-18 years with confirmed diagnosis of T1DM for at least one year were recruited consequently during their regular follow up visits at Pediatric Diabetes Outpatient Clinic at University Children's Hospital. The study was approved by the Dental Research Ethics Committee, Faculty of Dentistry, Institutional Research Board (Code no. A12140420).

Patients had chronic systemic comorbidities, receiving antibiotics over the previous 3 months, or currently receiving orthodontic treatment, were excluded. After comprehensive medical history and physical examination, only 88 out of 100 children with T1DM were eligible for the study. Written informed consent was obtained from parents of all children included in the study.

The following data were extracted from patients` medical files: age at onset and duration of diabetes, mean values of glycosylated hemoglobin (HbA1c) over that last year and the results of urinary albumin to creatinine ratio (UACR) in early morning urine sample that performed annually as a screen for diabetic nephropathy. Based on the results of at least two out of three UACRs over a 3-month span, children classified into normoalbuminuic (UACR $<30 \mathrm{mg} / \mathrm{g}$ creatinine) or microalbuminuric (UACR $=30-299 \mathrm{mg} / \mathrm{g}$ creatinine).

\section{Oral Health Assessment Methodology}

Oral health evaluation was carried visually by a professional dentist in a separate room at Pediatric Diabetes Outpatient Clinic. All of the following variables were estimated for each child in the following order; simplified oral hygiene index (OHI-S), gingival index (GI), decayed, missed and filled due to caries permanent teeth (DMFT), decayed and filled due to caries deciduous teeth (dft) and stimulated salivary flow rate (SSFR).

Oral health assessments required approximately 25 minutes for each subject. Details of oral health assessment methodology represented in the electronic supplementary file. 


\section{Infection control measures}

To avoid cross infection, disposable dental mirrors and probes were used for examination in addition to periodontal probe. Also, disposable containers and syringes were used for saliva sample collection. Personal protective barriers (white coat, gloves, mask and protective eye glasses) were taken during examination. Tight plastic bags and sharp containers were used for waste disposal.

\section{Consistency of examination}

Intra-examiner calibration was done by the researcher to reduce the intra-examiner variability.

The examiner was calibrated using a series of training sessions designed to standardize data collection technique to achieve good levels of intraexaminer reliability before starting the study. Ten children of about 6-18 years of age having different levels of caries were examined twice a week at Pediatric Dental Clinic at Faculty of Dentistry, on both dental chair and ordinary chair to detect the caries condition using dft and DMFT indices according to WHO criteria. The results were compared to determine the reliability. The reliability level was evaluated using Kappa statistics and it was more than 0.8 which indicated a high intra-examiner consistency.

\section{Simplified oral hygiene index (OHI-S)}

Comprises both simplified debris index (DI-S) and simplified calculus index (CI-S) (Supplementary table 1) ${ }^{8}$ Such indices calculate the extension of debris and calculus accordingly on the pre-determined exposed tooth surface. There are six index teeth $(16,11,26,36,31$ and 46). All index teeth were examined from the buccal surface except the two lower molars from the lingual surface. The missed index teeth were substituted by these deciduous teeth respectively $(55,51,65,75,71$ and 85). ${ }^{9}$ After scores recording, the index values were calculated. For each child, to obtain the OHI-S, both debris and calculus scores were totaled, divided by the number of examined surfaces and then combined together. The clinical levels of good oral hygiene for total OHI-S extends from 0-1.2, while fair oral hygiene extends from 1.3-3 and poor oral hygiene scores ranges from 3.1-6.

\section{Gingival index (GI) ${ }^{10}$}

For each child, six selected teeth had been assessed (16, 12, 24, 36, 32 and 44). Missed index teeth were substituted by these deciduous teeth respectively (55, 52, 64, 75, 72 and 84$).{ }^{11}$ Gingival tissues around each index tooth were examined from buccal, lingual, mesial, and distal aspects using a periodontal prob. Each surface took a score ranges from 0- 3 (Supplementary table 1). GI of each tooth was calculated by adding the four scores together and dividing them by the number of examined surfaces while, the total individual GI was calculated by dividing by the total number of the examined teeth. The scale of mild gingivitis extends from 0 to 1 , moderate gingivitis from 1.1 to 2 and severe gingivitis from 2.1 to 3 .

\section{Dental caries diagnosis and scoring}

Each tooth was examined using a dental mirror, probe and artificial source of light on ordinary chair. Due to unavailability of dental chair and difficulty in arranging patient appointments for examination at dental clinic, the following prerequisites were taken to decrease the risk of decreasing examination accuracy than those performed in dentist office; the patient sit in upright position with his/her head adjusted on the chair with the assistance of his/her parent according to the examined jaw; good artificial white light source was adjusted overhead and perpendicular on the examined teeth to decrease the shadowing effect also, magnified, concave, antifog dental mirrors were used to allow clear view of the examined site.

The tooth was observed by visual means as much as possible and only questionable small lesions were checked by using an explorer. The teeth 
surfaces were cleaned using cotton rolls and the dental probes were used if there is any doubt about presence of caries for removing dental plaque, soft deposits, and evaluation of pit and fissure sealants and for careful inspection of smooth surfaces. The tooth was considered sound if it shows no evidence of filling or decay.

The incipient caries was excluded because they cannot reliably diagnosed. The following deficient characteristics in the teeth were marked as sound in the absence of other significant criteria: chalky white patches, hard discolored or rough spots and dark, shiny, hard pitted areas of enamel.

Positive dental caries was recorded when the tooth surface has a cavity, undermined enamel, detectable soft floor, or temporary filling, remaining root due to caries and recurrent or secondary caries. Dental caries experience was determined by summation of decayed, missed and filled teeth due to caries in permanent teeth (DMFT) index and summation of decayed and filled teeth due to caries in deciduous teeth (dft) index separately, according to the WHO caries diagnostic criteria for epidemiological studies. ${ }^{12}$

\section{Stimulated salivary flow rate calculation (SSFR): ${ }^{13}$}

Saliva was obtained between 9 and 12 a.m. to prevent the influence of circadian rhythm. Participants were advised not to eat, drink anything but water, or chew gums one hour before sampling, so, it was delayed to the end of examination. Subjects were permitted to sit straight on the chair and rest for a few minutes.

Whole saliva was stimulated by chewing paraffin pellets for half a minute to soften it and the collected saliva during this period was swallowed or expectorated. Then children were requested to pour the accumulated saliva into clean graduated containers every minute for a total of five minutes, the tubes containing the saliva were left to stand for a few minutes to clear the foam, the amounts obtained were divided by 5 to yield SSFR expressed in $\mathrm{ml} / \mathrm{min}$ that considered normal if it was more than $1 \mathrm{ml} / \mathrm{min}$; low between $0.7-1 \mathrm{ml} / \mathrm{min}$; and very low if less than $0.7 \mathrm{ml} / \mathrm{min}$.

\section{Statistical analysis}

Data analysis was performed using SPSS version.22 (IBM, SPSS). Shapiro-Wilk test was used to determine the normality distribution of data. Chi-square test was used to compare the numbers and percentages of qualitative data. Parametric quantitative data were presented as means and standard deviations and nonparametric ones as medians with minimum and maximum. One-Way ANOVA and t-tests were used for parametric data while Kruskal- Wallis, Mann-Whitney and Onesample Wilcoxon Signed Rank tests were used for non-parametric data. Variables correlations were done by using Spearman rank correlation. A significant result was considered if $\mathrm{P}$-value $<0.05$.

\section{RESULTS}

Eighty-eight children and adolescents with T1DM were enrolled in the current study. General and diabetes-related characteristics of children with T1DM were displayed in table 1.

The median age of the subjects was 12 years (range: 6-17 years); 52\% were in childhood period (6-12 years) and $48 \%$ were in adolescence period (12-18 years). They were 37 male (42\%) and 51 female $(58 \%)$. The median duration of T1DM was 3 years (range: 1-13 years). The patients were divided into three groups based on T1DM duration; $1-2$ years $(n=25 ; 28 \%), 2-5$ years $(n=37 ; 42 \%)$, and greater than 5 years $(\mathrm{n}=26 ; 30 \%)$. Children with diabetes were nearly equally distributed among age $(\mathrm{P}=0.670)$, gender $(\mathrm{P}=0.136)$ and diabetes duration $(\mathrm{P}=0.221)$ groups.

The median age at onset of T1DM was 7.5 years (range: $2.5-16.5$ years). Patients were divided into three groups based on the age at T1DM onset in relation to timing of teeth eruption as follow; those with onset of diabetes before eruption of permanent 
teeth (before 6 years of age; $n=30$ ), those with onset of diabetes at the start of permanent teeth eruption (6-12 years of age; $n=51$ ), and those at the start of 2nd molar eruption (12-18 years of age; $n=7)$ with higher frequency of children was at mixed dentition stage $(\mathrm{P}=0.001)$.

The median HbA1c level was 9\% (range: 6.814), patients were divided into three subgroups based on level of glycemic control: good glycemic control (HbA1c: $<7.5 \%$; $=18$ ), moderate glycemic control (HbA1c: 7.5-9\%; $\mathrm{n}=31$ ), and poor glycemic control (HbA1c >9\%; $n=39)$; with higher frequency of children had moderate to poor glycemic control $(\mathrm{P}=0.022)$.

Based on the extracted UACR results, higher frequency of children had normoalbuminuria $(n=74$; $84 \%)$ vs. those with microalbuminuria $(n=14 ; 16 \%)$ $(\mathrm{P}<0.001)$
Evaluation of oral health status parameters including SSFR, OHI-S and GI among total study population revealed that; the median SSFR $(\mathrm{ml} /$ min) was 0.8 (range: 0.1-3) with significantly higher frequency of patients who had normal $(43.2 \%)$ or very low (39.8\%) SSFR compared to those with low (17\%) SSFR $(\mathrm{P}=0.005)$. The median OHI-S was 2.27 (range: 0-4.67) with significantly higher frequency of patients had fair oral hygiene $(70.5 \%)$ $(\mathrm{P}<0.001)$. The median GI was 1.38 (range: 0-2.88) with significantly higher frequency of patients had moderate gingivitis $(53.4 \%)(\mathrm{P}<0.001)$ (Table 2).

Table (3) represented the analysis of oral health status parameters including OHI-S, GI, DMFT, dft, and SSFR among the study subgroups. There were no significant differences in oral health parameters between male and female patients and between diabetic children had normoalbuminuria and those had microalbuminuria $(\mathrm{P}>0.05)$.

TABLE (1): Demographic and diabetes-related characteristics of the study subgroups

\begin{tabular}{|c|c|c|c|}
\hline & Frequency & $\begin{array}{c}\text { Test of } \\
\text { significance }\end{array}$ & P-value \\
\hline \multicolumn{4}{|l|}{ Sex } \\
\hline Male & $37(42)$ & \multirow{2}{*}{$X^{2}=2.227$} & \multirow{2}{*}{0.136} \\
\hline Female & $51(58)$ & & \\
\hline \multicolumn{4}{|l|}{ Age (years) } \\
\hline Childhood (6-12) & $46(52.3)$ & \multirow{2}{*}{$X^{2}=0.182$} & \multirow{2}{*}{0.670} \\
\hline Adolescence (12-18) & $42(47.7)$ & & \\
\hline \multicolumn{4}{|l|}{ Age at onset of T1DM } \\
\hline Before 6 years of age & $30(34)$ & \multirow{3}{*}{$X^{2}=33.023$} & \multirow{3}{*}{$<0.001 *$} \\
\hline $6-12$ years of age & $51(58)$ & & \\
\hline $12-18$ years of age & $7(8)$ & & \\
\hline \multicolumn{4}{|l|}{ Duration of T1DM (years) } \\
\hline $1-2$ years & $25(28.4)$ & \multirow{3}{*}{$X^{2}=3.023$} & \multirow{3}{*}{0.221} \\
\hline $2-5$ years & $37(42.1)$ & & \\
\hline$>5$ years & $26(29.5)$ & & \\
\hline \multicolumn{4}{|l|}{ HbA1c (\%) } \\
\hline Good glycemic control $(<7.5 \%)$ & $18(20.5)$ & \multirow{3}{*}{$X^{2}=7.659$} & \multirow{3}{*}{$0.022 *$} \\
\hline Moderate glycemic control (7.5-9\%) & $31(35.2)$ & & \\
\hline Poor glycemic control (> 9\%) & $39(44.3)$ & & \\
\hline \multicolumn{4}{|l|}{ Diabetic Nephropathy } \\
\hline Microalbuminuria (-) & $74(84.1)$ & \multirow{2}{*}{$X^{2}=40.909$} & \multirow{2}{*}{$<0.001^{*}$} \\
\hline Microalbuminuria (+) & $14(15.9)$ & & \\
\hline
\end{tabular}


Significantly higher OHI-S value was observed in children with T1DM duration 2-5 years compared to other groups $(\mathrm{P}=0.036)$. Significantly higher GI $(\mathrm{P}=0.019)$ was detected in adolescence period (12-18 years) compared to those in late childhood (6-12 years) $(\mathrm{P}=0.019)$. Significant lower SSFR was detected in late childhood group (6-12 years) compared to those in adolescence period (12-18 years) $(\mathrm{P}=0.038)$.

Significant increase in the prevalence of permanent dental caries (DMFT-index) was observed during adolescence period $(\mathrm{P}=0.001)$, in children with longer T1DM duration ( $>5$ years) compared to those with diabetes duration $0.5-2$ years $(\mathrm{P}=0.019)$ and in children with poor compared to those with good glycemic control $(\mathrm{P}=0.028)$.

Significant increase in the prevalence of primary dental caries (dft-index) was observed in children with earlier onset of T1DM (before six years of age) compared to those with age at onset of T1DM between 6-12 years $(\mathrm{P}=0.004)$ and in children with good compared to those with poor glycemic control $(\mathrm{P}=0.010)$.

Correlation analyses revealed that patient's age was positively correlated with SSFR, DMFT, and GI. There were significant positive correlation between GI and age at T1DM onset and between DMFT and both T1DM duration and HbA1c level. The dftindex was negatively correlated with patient's age, age at onset and duration of T1DM, and HbA1c level. Among oral health-related variables, there was significant positive correlation between OHI-S and GI and significant negative correlation between dft-index and both SSFR and DMFT (Table 4).

TABLE (2): Oral health-related characteristics of the study population

\begin{tabular}{|c|c|c|c|c|}
\hline & & Frequency & Test of significance & P-value \\
\hline $\mathrm{SSFR}(\mathrm{ml} / \mathrm{min})$ & & & & \\
\hline Normal & $(\geq 1)$ & $38(43.2)$ & \multirow{3}{*}{$X^{2}=10.659$} & \multirow{3}{*}{$0.005 *$} \\
\hline Low & $(0.7-1)$ & $15(17)$ & & \\
\hline Very low & $(<0.7)$ & $35(39.8)$ & & \\
\hline \multicolumn{5}{|l|}{ OHI-S } \\
\hline Good oral hygiene & $(0-1.2)$ & $18(20.5)$ & \multirow{3}{*}{$X^{2}=56.273$} & \multirow{3}{*}{$<0.001^{*}$} \\
\hline Fair oral hygiene & $(1.3-3)$ & $62(70.5)$ & & \\
\hline Poor oral hygiene & $(3.1-6)$ & $8(9)$ & & \\
\hline \multicolumn{5}{|l|}{ GI } \\
\hline Mild gingivitis & $(0-1)$ & $30(34.1)$ & \multirow[t]{3}{*}{$X^{2}=22.114$} & \multirow[t]{3}{*}{$<0.001^{*}$} \\
\hline Moderate gingivitis & $(1.1-2)$ & $47(53.4)$ & & \\
\hline Severe gingivitis & $(2.1-3)$ & $11(12.5)$ & & \\
\hline
\end{tabular}

Data presented as number (\%)

*statistically significant difference

GI, gingival index; OHI-S, simplified oral hygiene index; SSFR, stimulated salivary flow rate. 
TABLE (3): Oral health status parameters among the study subgroups:

\begin{tabular}{|c|c|c|c|c|c|c|}
\hline & & OHI-S & GI & dft & DMFT & SSFR \\
\hline \multirow[t]{3}{*}{ Age (year) } & $6-12$ years & $2(0.5-4.67)$ & $1.3 \pm .53$ & $2(0-16)$ & $0(0-5)$ & $0.6(0.2-2)$ \\
\hline & $12-18$ years & $2.5(0-4.5)$ & $1.6 \pm .57$ & & $2(0-10)$ & $1(0.1-3)$ \\
\hline & P-value & 0.105 & $0.019 *$ & 0.167 & $0.001^{*}$ & $0.038 *$ \\
\hline \multirow[t]{3}{*}{ Sex } & Male & $2(0-3.67)$ & $1.13(0-2.54)$ & $0(0-16)$ & $1(0-10)$ & $1(0.2-3)$ \\
\hline & Female & $2.3(0.5-4.67)$ & $1.46(0.75-2.88)$ & $0(0-10)$ & $2(0-10)$ & $0.8(0.1-2.4)$ \\
\hline & P-value & 0.324 & 0.212 & 0.357 & 0.148 & 0.159 \\
\hline \multirow{4}{*}{$\begin{array}{l}\text { Age at onset } \\
\text { of T1DM }\end{array}$} & G1 (before 6 years) & $2(0.5-4.67)$ & $1.25(0-2.58)$ & $1(0-16)$ & $0.5(0-10)$ & $0.7(0.2-3)$ \\
\hline & G2 (6-12 years) & $2.33(0-4.5)$ & $1.38(0.25-2.54)$ & $0(0-6)$ & $1(0-8)$ & $0.8(0.1-2.4)$ \\
\hline & G3 (12-18 years) & $3(1-3.83)$ & $1.75(1-2.88)$ & & $1(0-10)$ & $1(0.3-2.2)$ \\
\hline & P-value & 0.233 & 0.186 & $0.004 *$ & 0.932 & 0.334 \\
\hline \multirow{4}{*}{$\begin{array}{l}\text { Duration of } \\
\text { T1DM }\end{array}$} & G1 (1-2 years) & $2(1-3.83)$ & $1.37 \pm .54$ & $0(0-16)$ & $0(0-5)$ & $0.7(0.2-2)$ \\
\hline & G2 (2-5 years) & $2.67(0.67-4.67)$ & $1.53 \pm .58$ & $0(0-10)$ & $1(0-10)$ & $0.9(0.1-2.4)$ \\
\hline & G3 (>5 years) & $2.09(0-3.33)$ & $1.40 \pm .56$ & $0(0-10)$ & $2.5(0-10)$ & $0.8(0.3-3)$ \\
\hline & $P$-value & $0.036^{* a, c}$ & 0.463 & 0.148 & $0.019 * \mathrm{~b}$ & 0.325 \\
\hline \multirow[t]{4}{*}{$\mathrm{HbAlc} \%$} & G1 $(<7.5 \%)$ & $2.25(0.67-4.67)$ & $1.5(.75-2.58)$ & $2(0-16)$ & $0(0-9)$ & $0.6(0.2-2)$ \\
\hline & G2 (7.5-9\%) & $2.5(0-3.83)$ & $1.25(0-2.88)$ & $0(0-10)$ & $1(0-10)$ & $0.9(0.1-2.4)$ \\
\hline & $\mathrm{G} 3(>9 \%)$ & $2.17(0.71-4.5)$ & $1.5(0.75-2.54)$ & $0(0-6)$ & $2(0-10)$ & $0.8(0.2-3)$ \\
\hline & $P$-value & 0.895 & 0.464 & $0.010^{* \mathrm{~b}}$ & $0.028 * \mathrm{~b}$ & 0.374 \\
\hline \multirow{3}{*}{$\begin{array}{l}\text { Diabetic } \\
\text { Nephropathy }\end{array}$} & Microalbumine (-) & $2(0-4.67)$ & $1.38(0-2.88)$ & $0(0-16)$ & $1(0-10)$ & $0.8(0.1-2.4)$ \\
\hline & Microalbumine (+) & $2.59(1-3.33)$ & $1.75(0.75-1.92)$ & $0(0-6)$ & $1(0-10)$ & $0.85(0.2-3)$ \\
\hline & $P$-value & 0.133 & 0.761 & 0.119 & 0.389 & 0.950 \\
\hline
\end{tabular}

Data presented as mean $\pm S D$ or as median (min-max)

DMFT, decayed, missed and filled due to caries permanent teeth; dft, decayed and filled due to caries deciduous teeth; GI, gingival index; OHI-S, simplified oral hygiene index; SSFR, stimulated salivary flow rate.

*statistically significant difference

a, Significant difference between group 1 (G1) and group 2 (G2)

b, Significant difference between group 1 (G1) and group 3 (G3)

c, Significant difference between group 2 (G2) and group 3 (G3) 
TABLE (4): Spearman's correlation analysis between diabetes-related variables and oral health-related variables of the study population

\begin{tabular}{|c|c|c|c|c|c|c|}
\hline & & SSFR & DMFT & $\mathrm{dft}$ & OHIS & GI \\
\hline \multirow[t]{2}{*}{ Age } & $\mathrm{r}$ & $0.317^{*}$ & $0.466^{*}$ & $-0.776^{*}$ & 0.195 & $0.216^{*}$ \\
\hline & $\mathrm{P}$ & 0.003 & $<0.001$ & $<0.001$ & 0.069 & 0.043 \\
\hline \multirow[t]{2}{*}{ Age at T1DM onset } & $\mathrm{r}$ & 0.141 & 0.093 & $-0.484^{*}$ & 0.147 & $0.212^{*}$ \\
\hline & $\mathrm{P}$ & 0.190 & 0.391 & $<0.001$ & 0.172 & 0.048 \\
\hline \multirow[t]{2}{*}{ Duration of T1DM } & $\mathrm{r}$ & 0.136 & $0.355^{*}$ & $-0.276^{*}$ & 0.031 & 0.023 \\
\hline & $\mathrm{P}$ & 0.205 & 0.001 & 0.009 & 0.774 & 0.833 \\
\hline \multirow[t]{2}{*}{ HbAlc $(\%)$} & $\mathrm{r}$ & -0.136 & $0.254^{*}$ & $-0.275^{*}$ & -0.035 & 0.073 \\
\hline & $\mathrm{P}$ & 0.206 & 0.017 & 0.010 & 0.743 & 0.497 \\
\hline \multirow[t]{2}{*}{ SSFR } & $\mathrm{r}$ & & 0.108 & $-0.278^{*}$ & -0.007 & 0.037 \\
\hline & $\mathrm{P}$ & & 0.316 & 0.009 & 0.951 & 0.736 \\
\hline \multirow[t]{2}{*}{ DMFT } & $\mathrm{r}$ & & & $-0.357^{*}$ & 0.117 & 0.105 \\
\hline & $\mathrm{P}$ & & & 0.001 & 0.276 & 0.331 \\
\hline \multirow[t]{2}{*}{$\mathrm{dft}$} & $\mathrm{r}$ & & & & -0.145 & -0.141 \\
\hline & $\mathrm{P}$ & & & & 0.177 & 0.189 \\
\hline \multirow[t]{2}{*}{ OHIS } & $\mathrm{r}$ & & & & & $0.453^{*}$ \\
\hline & $\mathrm{P}$ & & & & & $<0.001$ \\
\hline
\end{tabular}

DMFT, decayed, missed and filled due to caries permanent teeth; dft, decayed and filled due to caries deciduous teeth; GI, gingival index; OHI-S, simplified oral hygiene index; SSFR, stimulated salivary flow rate.

*statistically significant difference

\section{DISCUSSION}

A bidirectional relationship between T1DM and oral health status has been proposed by several studies, where T1DM has a considerable role in the initiation and progression of oral diseases including; periodontitis, dental caries, and even can cause changes of the cariogenic oral microbiome composition $^{14}$, and on the other hand, periodontitis causes difficulties in glycemic control among children with T1DM..$^{15}$

Oral health has been demonstrated to influence the physical, psychological, and social life aspects of children and adolescents with T1DM. In a recent study, oral health-related quality of life parameters were adversely affected by T1DM particularly in children with poor glycemic control. ${ }^{16}$

The results of this study demonstrated that oral health status were influenced by diabetes-related factors such as age at onset and duration of T1DM and the level of metabolic control, where low SSFR, fair oral hygiene, moderate gingivitis and dental caries of deciduous and permanent teeth were detected among different T1DM subgroups.

Salivary flow serves as an oral health defense agent. Therefore, a decrease in the salivary flow is associated with an increase in the risk of oral diseases including dental caries. ${ }^{17}$ In the current study, we observed a significantly lower SSFR in 
children within late childhood group compared to adolescence group with positive association between SSFR and the age, this can be explained by increased SFR with age because of increasing mechanical receptors stimulation and salivary glands seemed to be fully developed at the age of $15^{13}$, regardless of the groups distribution which trends to patients with abnormal glycemic control (80\%). In addition, lower SSFR was observed in children with earlier T1DM onset although not reaching a significant level.

Several mechanisms have been postulated to underlie the lower SSFR among patients with DM, of these the adverse effect of decreased extracellular fluid secondary to diuresis on salivary flow, as well as the impairment of autonomic regulation that adversely affect the response of salivary glands to neural or hormonal stimuli. Our results agree with the results reported by Ferizi et al, ${ }^{18}$ who found a significantly lower SSFR in children with T1DM compared to healthy controls.

However, we did not detect a significant association between SSFR and level of glycemic control. In agreement, the absence of such association reported previously, ${ }^{19}$ this can be related to the smaller sample size and overall quite high HbA1c level.

We identified that primary dental caries was significant in children who were diagnosed with diabetes before six years, also we demonstrated an increased prevalence of dental caries of permanent teeth in the late adolescence period, in children with prolonged T1DM duration (more than five years), and in those with poor glycemic control $(\mathrm{HbAlc}$ $>9 \%$ ). This can be attributed to the salivary gland hypofunction and decrease in salivary flow rate and high glucose concentrations in saliva that can be found in poorly controlled T1DM patients. In addition, improper oral hygiene with accumulated plaque may has a great role in the development of dental caries. ${ }^{20}$
In our study the high prevalence of dental caries might be related to the observed significant positive correlation between DMFT and HbA1c level. Poor glycemic control might lead to diminished salivary flow rate and impaired salivary buffer capacity that in turn enhance the increase of cariogenic bacteria. Moreover, high levels of $\mathrm{HbA} 1 \mathrm{c}$ usually reflect poor dietary habits with higher sugary diet intake. ${ }^{21}$

Our findings are consistent with the majority of studies which had identified higher caries rates in individuals with poorly controlled T1DM when compared to those with good glycemic control..$^{22}$ In addition, Twetman et al. ${ }^{23}$ reported a higher rate of dental caries in children (8-16 years) with T1DM with poor glycemic control $(\mathrm{HbA} 1 \mathrm{c}>8 \%)$, and confirmed that those patients had a higher salivary glucose concentration. On the other hand, absence of an association between $\mathrm{HbAlc}$ and caries rate in children with T1DM has been reported by several previous studies. ${ }^{24}$

In the current study, OHI-S in all groups was considered fair, and it was not associated with $\mathrm{HbA} 1 \mathrm{c}$ levels. This lies in line with the results of a study carried out by Wyne and colleagues to explore OHI-S in 134 children with T1DM and found that most of patients had fair OHI-S index..$^{25}$

The majority of enrolled children in the current study had moderate gingivitis with significant association between GI and patients`age, that may be attributed to improper oral hygiene as plaque is the precursor of gingivitis. Moreover, gingiva appears to be a target organ for sex steroids at puberty. These results are in line with previous studies which consistently reported greater prevalence of gingivitis in T1DM patients when compared to nondiabetic control. ${ }^{26}$

In the current study, there were no significant differences in oral health parameters between diabetic children with microalbuminuria and those with who are normoalbuminuric, however, most of the enrolled children were normoalbuminuric (84\%). In previous studies conducted on 207 Indian adults 
with T2DM, periodontitis was associated with poor metabolic control and with diabetic nephropathy. ${ }^{27}$

Based on the results of correlation analyses, we observed that SSFR positively correlate with both age and age of diabetes onset. ${ }^{22}$ DMFT, was also positively correlated with age and this could be referred to the increased number of susceptible erupted permanent teeth, in addition the permanent dental caries experience was affected by poorer metabolic control and increased duration of diabetes. ${ }^{22}$ On the other hand, a negative correlation between $\mathrm{dft}$ and age of children with T1DM was found; this can be explained by the decreased number of primary teeth by aging due to shedding. This is coinciding with a recent cross-sectional study, conducted by Abbas et $\mathrm{al}^{28}$ to detect the prevalence of dental caries regarding age, gender and concluded that, in mixed dentition, dft was negatively correlated with age while, in permanent dentition, DMFT in children was positively correlated with age.

Also, dft showed a negative correlation with other diabetes-related and oral health-related variables and this is mainly due to differences in group frequencies especially onset of diabetes which significantly start at six years. ${ }^{29}$ Finally, the gingival severity stage increased with poor oral hygiene and earlier onset of T1DM. ${ }^{30}$ All previous correlations point to the considerable impact of the diabetesrelated factors including age of onset and duration of T1DM as well as level of metabolic control on the development and progression of oral diseases as recently suggested by Babatzia et al. ${ }^{31}$

The observed associations between OHI-S, GI, and dental caries is linked to that they share similar behaviors, i.e. inadequate oral hygiene practices and unhealthy dietary intake, where insufficient tooth brushing and excess sugary foods intake may result in greater detrimental oral effects. ${ }^{3}$ The observed correlations between the patient's age and SSFR, DMFT, dft and GI highlight the importance of early counseling for oral health at the onset of T1DM and reinforce the importance of thereafter regular dental visits, once children with T1DM represent a high risk population for adverse oral health.

\section{CONCLUSION}

Children with T1DM displayed unfavorable oral health profiles including fair oral hygiene, moderate gingivitis, increased prevalence of both primary and permanent dental caries, and low SSFR that have been strongly linked to patient's age, age at onset and duration of T1DM and level of metabolic control. Care of children with T1DM requires a multidisciplinary team including dental health professionals. Regular dental visits for oral health checkups rather than dental visits on needs must be considered in the routine care of children with diabetes. Further longitudinal oral health studies involving larger sample size of children with T1DM need to be approved and carried out.

\section{ACKNOWLEDGEMENT}

We are grateful to all the families that participated in this study.

\section{REFERENCES}

1. Edition I. International Diabetes Federation. IDF Diabetes Atlas, 8th edn. Brussels, Belgium: International Diabetes Federation, 2017.

2. LM CJKML, Peters A. Type 1 diabetes through the life span: a position statement of the American Diabetes Association. Diabetes Care 37: 2034-2054, 2014.

3. Lifshitz F, Casavalle P, Bordoni N, Rodriguez P, Friedman $S$. Oral Health in Children with Obesity or Diabetes Mellitus. Pediatric endocrinology reviews: PER 14(2): 159-167, 2016.

4. Frantzis TG, Reeve CM, Brown Jr AL. The ultrastructure of capillary basement membranes in the attached gingiva of diabetic and nondiabetic patients with periodontal disease. Journal of periodontology 42(7): 406-411, 1971.

5. Leite RS, Marlow NM, Fernandes JK, Hermayer K. Oral health and type 2 diabetes. The American journal of the medical sciences 345(4): 271-273, 2013. 
6. Teeuw WJ, Gerdes VE, Loos BG. Effect of periodontal treatment on glycemic control of diabetic patients: a systematic review and meta-analysis. Diabetes care. 33(2): 421-427, 2010.

7. Sim J, Wright CC. The kappa statistic in reliability studies: use, interpretation, and sample size requirements. Physical therapy 85(3): 257-268, 2005.

8. Greene JG, Vermillion JR. The simplified oral hygiene index. The Journal of the American Dental Association 68(1): 7-13, 1964.

9. Rodrigues C, Ando T, Guimaraes L. Simplified oral hygiene index for ages 4 to 6 and 7 to 10 (deciduous and mixed dentition). Revista de odontologia da Universidade de Sao Paulo 4(1): 20-24, 1990.

10. Silness J, Löe H. Periodontal disease in pregnancy II. Correlation between oral hygiene and periodontal condition. Acta odontologica scandinavica 22(1):121-135, 1964.

11. Goldberg P, Matsson L, Anderson H. Partial recording of gingivitis and dental plaque in children of different ages and in young adults. Community dentistry and oral epidemiology 13(1): 44-46, 1985.

12. Organization WH. WHO basic oral health survey. Santos, São Paulo 4, 1997.

13. Falcão DP, da Mota LMH, Pires AL, Bezerra ACB. Sialometry: aspects of clinical interest. Revista Brasileira de Reumatologia (English Edition) 53(6): 525-531, 2013.

14. Arheiam A, Omar S. Dental caries experience and periodontal treatment needs of 10-to 15 -year old children with type 1 diabetes mellitus. International dental journal 64(3): 150-154, 2014.

15. Mirza B, Syed A, Izhar F, Ali Khan A. Bidirectional relationship between diabetes and periodontal disease: review of evidence. J Pak Med Assoc 60(9): 766-768, 2010 ..

16. Elheeny AAH. Oral health status and impact on the oral health-related quality of life of Egyptian children and early adolescents with type- 1 diabetes: a case-control study. Clin Oral Investig 2020.

17. Cunha-Cruz J, Scott J, Rothen M, et al. Salivary characteristics and dental caries: evidence from general dental practices. J Am Dent Assoc 144(5): e31-40, 2013.
18. Ferizi L, Dragidella F, Spahiu L, Begzati A, Kotori V. The influence of type 1 diabetes mellitus on dental caries and salivary composition. International journal of dentistry. $2018,2018$.

19. Javed F, Sundin U, Altamash M, Klinge B, ENGSTRÖM PE. Self-perceived oral health and salivary proteins in children with type 1 diabetes. Journal of oral rehabilitation 36(1): 39-44, 2009.

20. Mussavira S, Dharmalingam M, Sukumaran BO. Salivary glucose and antioxidant defense markers in type II diabetes mellitus. Turkish journal of medical sciences 45(1): 141$147,2015$.

21. Wang Y, Xing L, Yu H, Zhao L. Prevalence of dental caries in children and adolescents with type 1 diabetes: a systematic review and meta-analysis. BMC oral health 19(1): 213, 2019.

22. Carneiro VL, Fraiz FC, Ferreira FdM, Pintarelli TP, Oliveira ACB, Boguszewski MCdS. The influence of glycemic control on the oral health of children and adolescents with diabetes mellitus type 1 . Archives of endocrinology and metabolism 59(6): 535-540, 2015.

23. Twetman S, Johansson I, Birkhed D, Nederfors T. Caries incidence in young type 1 diabetes mellitus patients in relation to metabolic control and caries-associated risk factors. Caries research 36(1): 31-35, 2002.

24. Busato IMS, Bittencourt MS, Machado MÂN, Grégio AMT, Azevedo-Alanis LR. Association between metabolic control and oral health in adolescents with type 1 diabetes mellitus. Oral Surgery, Oral Medicine, Oral Pathology, Oral Radiology, and Endodontology 109(3): e51-e56, 2010.

25. Wyne AH, Chohan AN, Al-Sharari R. Caries, Oral Hygiene and Gingival Health Status in Type 1 Diabetic Saudi Children. Pakistan Oral \& Dental Journal 36(3), 2016.

26. Cutler CW, Machen RL, Jotwani R, Iacopino AM. Heightened gingival inflammation and attachment loss in type 2 diabetics with hyperlipidemia. Journal of periodontology 70(11): 1313-1321, 1999.

27. Khanuja PK, Narula SC, Rajput R, Sharma RK, Tewari S. Association of periodontal disease with glycemic control in patients with type 2 diabetes in Indian population. Frontiers of medicine 11(1): 110-119, 2017.

28. Abbass MM, Mahmoud SA, El Moshy S, et al. The prevalence of dental caries among Egyptian children and 
adolescences and its association with age, socioeconomic status, dietary habits and other risk factors. A crosssectional study. F1000Research 8, 2019.

29. Ismail AF, McGrath CP, Yiu CK. Oral health of children with type 1 diabetes mellitus: A systematic review. Diabetes research and clinical practice 108(3): 369-381, 2015.
30. Newman MG, Takei H, Klokkevold PR, Carranza FA. Carranza's clinical periodontology. Elsevier health sciences; 2011.

31. Babatzia A, Papaioannou W, Stavropoulou A, et al. Clinical and microbial oral health status in children and adolescents with type 1 diabetes mellitus. International dental journal 70(2): 136-144, 2020. 